\title{
Factores de Riesgo Psicosocial y Burnout en Población Económicamente Activa de Lima, Perú
}

\author{
PSYCHOSOCIAL RISK FACTORS AND BURNOUT IN ECONOMICALLY ACTIVE POPULATION OF LIMA, PERU
}

\author{
Ma Del Refugio López Palomar', Sara Adriana García Cueva1', Manuel Pando Moreno² \\ 1. CU de los Valles, Universidad de Guadalajara, México. \\ 2. Instituto de Investigación en Salud Ocupacional, Universidad de Guadalajara, México.
}

\begin{abstract}
RESUMEN
El estudio de los factores de riesgo psicosocial recobra mayor relevancia ante las dinámicas impuestas por las economías globalizadas que generan entornos competitivos, aumentando las exigencias físicas, psicológicas y sociales de los trabajadores, quienes manifiestan diversas respuestas como el estrés laboral crónico también conocido como burnout o Síndrome de Quemarse por el Trabajo (SQT).

El presente estudio tuvo como objetivo determinar la prevalencia del burnout y su relación con la presencia de factores de riesgo psicosocial laborales, percibidos como negativos en trabajadores de la Población Económicamente Activa (PEA) de Lima, Perú. Se encuestaron 339 trabajadores con la aplicación del Inventory General Survey (MBI-GS) ${ }^{1}$, instrumento en su nueva versión, y la escala de Factores Psicosociales en el Trabajo ${ }^{2}$, encontrándose prevalencia de burnout muy alto en la dimensión desgaste emocional o agotamiento $(6,22 \%)$, asociado con 4 diferentes factores de riesgo psicosocial. El mayor factor de riesgo lo representan las exigencias laborales $(p<0,004)$ y $(O R=6,979)$ con la dimensión cinismo de burnout; lo anterior deja de manifiesto que ante las exigencias laborales, los trabajadores expresan actitudes cínicas como mecanismo de defensa, por lo que se concluye que la prevalencia del burnout se relaciona significativamente con los factores de riesgo psicosocial, de ahí que las organizaciones deben prestar especial atención en estos factores.
\end{abstract}

(López M, García S, Pando M, 2014. Factores de Riesgo Psicosocial y Burnout en Población Económicamente Activa de Lima, Perú. Cienc Trab. Sep-Dic; 16 [51]: 164-169).

Palabras clave: BURNOUT, MASLACH BURNOUT INVENTORYGENERAL SURVEY (MBI-GS), FACTORES PSICOSOCIALES, RIESGO PSICOSOCIAL, POBLACIÓN ECONÓMICAMENTE ACTIVA, LIMA, PERÚ.

\section{ABSTRACT}

The study of psychosocial risk factors regains more relevance to the dynamics imposed by globalized economies that generate competitive environments, increasing the physical, psychological and social needs of workers who manifest different answers as chronic job stress also known as Burnout or Syndrome of burning for Work (SQT).

This study aimed to determine the prevalence of burnout and its relationship with the presence of occupational psychosocial risk factors, perceived as negative in workers of the Economically Active Population (EAP) in Lima, Peru. 339 workers were surveyed with the implementation of Inventory General Survey (MBI-GS) ${ }^{1}$, instrument in its new version, and the scale of Psychosocial Factors in Work ${ }^{2}$, finding high prevalence of burnout in the dimension emotional exhaustion $(6,22 \%)$, associated with 4 different psychosocial risk factors, the biggest risk factor is represented by work demands ( $p$ $<0,004)$ and $(\mathrm{OR}=6,979)$ with the cynicism of burnout dimension, the previous makes it clear that with work demands, workers expressed cynicism as a defense mechanism, so it is concluded that the prevalence of burnout was significantly related to psychosocial risk factors, hence that organizations should pay special attention to these factors.

Key words: Burnout. MASLACH BURNOUT INVENTORY-GENERAL SURVEY (MBI-GS), PSYCHOSOCIAL FACTORS, PSYCHOSOCIAL RISK, ECONOMICALLY ACTIVE POPULATION, LIMA PERU.

\section{INTRODUCCIÓN}

Los Factores de Riesgo Psicosocial en el Trabajo se han convertido en uno de los temas de mayor preocupación entre los diversos actores involucrados en el ámbito de las organizaciones, la salud, seguridad e higiene ocupacional. Los riesgos o factores de riesgo

Correspondencia / Correspondence:

Ma Del Refugio López Palomar

Juárez No. 58 en San Martín Hidalgo, Jalisco, México

Tel.: 0453313472116

e-mail: cuquis@profesores.valles.udg.mx

Recibido: 20 de Agosto 2014 / Aceptado: 24 de Octubre 2014 psicosociales se han definido por la Organización Internacional de Trabajo, $\mathrm{OIT}^{3}$, como:

"Las interacciones entre el contenido, la organización y la gestión del trabajo y las condiciones ambientales, por un lado, y las funciones y necesidades de los trabajadores, por otro. Estas interacciones podrian ejercer una influencia nociva en la salud de los trabajadores a través de sus percepciones y experiencia”.

Por su parte, la Agencia Europea de Salud y Seguridad en el Trabajo EU-OSHA 4 , establece que por "factor de riesgo" de origen psicosocial se entiende todo "aspecto de la concepción, organización y gestión del trabajo así como de su contexto social y ambiental que tiene la potencialidad de causar daños físicos, sociales o psicológicos en los trabajadores".

Diversas investigaciones señalan que evaluar los riesgos psicosociales y sus consecuencias es esencial por el impacto que tienen en la salud física y psicológica del trabajador, su satisfacción, su calidad de vida, su productividad, etc. ${ }^{5}$ 
Marrero, Román y Salomón ${ }^{6}$ manifiestan que el estrés laboral es considerado como respuesta nociva que ocurre cuando las exigencias del trabajo rebasan las capacidades y los recursos del trabajador, llegando a convertirse en uno de los principales efectos negativos del trabajo y se constituye un riesgo psicosocial laboral. El sindrome de burnout surge por la exposición crónica a factores de riesgo de tipo psicosocial, se presenta como una manifestación o respuesta al estrés crónico laboral y se caracteriza por tres dimensiones: a) agotamiento emocional (agotamiento de los recursos emocionales propios); b) despersonalización (actitudes negativas), y c) falta de realización personal en el trabajo (valoración negativa del propio rol profesional) ${ }^{7}$.

Para estudiar el burnout se han desarrollado dos perspectivas, la clínica y la psicosocial. Desde la perspectiva clínica, Freudenberger ${ }^{8}$ empleó por vez primera el término burnout para describir un conjunto de sintomas físicos sufridos por personal sanitario como resultado de las condiciones de trabajo. Lo definió como fallar, agotarse, o llegar a desgastarse debido a un exceso de fuerza, demandas excesivas de energía o de recursos, señalando lo que ocurre cuando un profesional de servicios de ayuda "se quema" y fracasa en alcanzar sus objetivos; y se caracteriza por un estado de agotamiento como consecuencia de trabajar intensamente, sin tomar en consideración las propias necesidades.

La perspectiva psicosocial considera al burnout un proceso dinámico; la definición más aceptada es la elaborada por Maslach y Jackson, quienes lo consideran como una respuesta, principalmente emocional, situando los factores laborales y los organizacionales como condicionantes y antecedentes. Los estudios defienden que burnout es un sindrome tridimensional que se desarrolla en aquellos profesionales cuyo objeto de trabajo son personas (usuarios) y añaden tres dimensiones características: el Agotamiento emocional, Despersonalización y Bajo Logro o Realización Profesional y/o Personal. ${ }^{9-11}$

Desde la perspectiva psicosocial el síndrome de burnout se entiende como un proceso que desarrolla secuencialmente la aparición de sus rasgos y sus síntomas globales. Algunos autores van a discrepar en el síntoma que se presenta en primer lugar, la secuencia del proceso, el protagonismo que conceden a cada uno de sus sintomas globales, en la explicación de los mecanismos y en las estrategias que el individuo puede emplear para manejarlo. Lo anterior da origen al surgimiento de varios modelos procesuales que tratan de explicar su desarrollo, como lo son los modelos etiológicos explicativos del síndrome, quedado estructurado en tres grandes teorías: socio-cognitiva, teoría del intercambio social y la teoría organizacional. El presente estudio se aborda desde la perspectiva psicosocial.

Los estudios iniciales del burnout, se centraron en profesiones y ocupaciones asistenciales, por ello fue conceptualizado como efecto de la interacción con los usuarios de servicios, principalmente asistenciales. De acuerdo a diversos estudios realizados, el burnout no se reduce a campos asistenciales, ya que en numerosas profesiones y ocupaciones se presenta como resultado de estrés crónico, básicamente laboral; el burnout se ha propuesto incluso para actividades no laborales tales como las actividades parentales o para relaciones maritales. ${ }^{12,13}$

En este sentido, Maslach, Jakson y Leiter redefinen el burnout como "una crisis en la relación con el propio trabajo, sin que signifique necesariamente una crisis de las relaciones con las personas en el trabajo". La redefinición antes expresada dio como resultado el Maslach Burnout Inventory-General Survey (MBI-GS), que consta de 16 items, y es una adaptación del cuestionario clásico (MBI-Human Services Survey de 22 items), utilizado para profesionales de la salud. ${ }^{14}$

Cuando se evalúa el Síndrome de Burnout mediante el MBI-GS se está valorando una crisis en la relación de una persona con su trabajo, y no necesariamente una crisis entre una persona y la gente con la que se relaciona esta. Por lo anterior el MBI-GS es propuesto como un instrumento para evaluar las actitudes ante el propio trabajo y las escalas propuestas son las siguientes:

Desgaste Emocional (5 items): Se define de forma genérica, sin el énfasis en los aspectos emocionales acerca de los "recipientes del propio trabajo" presentes en el MBI-HSS. Todos los ítems provienen del MBI-HSS, modificados o sin modificar.

Cinismo (5 items): Refleja la actitud de indiferencia, devaluación y distanciamiento ante el propio trabajo, así como el valor y significación que se le pueda conceder, representa igualmente una actitud defensiva ante las agotadoras demandas provenientes del trabajo.

Eficacia profesional (6 ítems): está relacionada con las expectativas que se tienen en relación con la profesión que se desempeña, y se expresa en las creencias del sujeto sobre su capacidad de trabajo, su contribución eficaz a la organización laboral, de haber realizado cosas que realmente valen la pena y de realización profesional, semejante a la anterior de realización personal pero insistiendo en las expectativas según el modelo de Bandura. ${ }^{15} \mathrm{La}$ redefinición del burnout atiende a la identificación de este como un problema de salud derivado de la persona con su trabajo.

La estructura factorial de la adaptación al castellano del Maslach Burnout Inventory-General Survey (MBI-GS) realizada por Gil-Monte ${ }^{16}$ reporta valores de fiabilidad según alfa de Cronbach, encontrándose 0,85 para Eficacia profesional, 0,83 para Agotamiento y 0,74 para cinismo, similares a lo reportado por Moreno, Rodríguez y Escobar ${ }^{14}$ cuyos valores oscilan entre 0,84 y/o 0,89 para las tres subescalas, por encima de los índices de consistencia presentados en el manual del MBI-GS que están en los 0,73 a 0,89 (Maslach, Jackson $\mathrm{y}$ Leiter). Por lo anterior el cuestionario se considera válido $\mathrm{y}$ confiable en la versión adaptada al castellano.

Algunos estudios refieren que los factores de riesgo psicosocial a los que están expuestos los trabajadores sin diferenciar la profesión y el nivel de estudios, pueden ocasionar desde mínimas consecuencias en la salud hasta la muerte. ${ }^{17}$

La OIT ${ }^{18}$ ratifica en el informe 2013 sobre el Día Mundial de la Seguridad y Salud en el Trabajo que el burnout no es exclusivo de profesiones asistenciales. Resalta que los cambios tecnológicos, sociales y organizativos dan origen a nuevas enfermedades profesionales vinculadas con el estrés laboral que empobrecen la salud de los trabajadores, sus familias y las empresas al reducir la productividad y la capacidad de trabajo; en este sentido la OIT ${ }^{19}$, en la guía del formador SOLVE, destaca el impacto de los factores psicosociales en el trabajo y su vinculación con el estrés, burnout y el acoso psicológico (mobbing), actualmente considerados como problemas globales presentes en todas las profesiones y ocupaciones, en países desarrollados y no desarrollados, convirtiéndose en amenazas importantes a la supervivencia de una empresa.

En Perú la legislación ha avanzado en la materia de Prevención de Riesgos Laborales (PRL) o riesgos psicosociales como lo manifiestan en su análisis Raffo, Ráez y Cachay $^{20}$, al señalar que las leyes de Seguridad y Salud en el Trabajo en este país promueven una cultura de la PRL, resaltando que los artículos de las leyes en 
la materia presentan buenas intenciones, sin embargo se adolece de anexos y guías metodológicas de cómo actuar en la identificación y la evaluación de los riesgos psicosociales.

Fernández Arata $^{21}$, realizó un estudio sobre Burnout, Autoeficacia y Estrés en maestros de escuelas primarias y secundarias de Lima, Perú, reportando la presencia de Burnout elevado, manifestando que fue más alto en los docentes de escuela primaria $(43,2 \%)$ que en los de secundaria $(36,6 \%)$. Encontró una relación significativa entre los estresores y el burnout.

Datos relevantes ofrecen los resultado de la encuesta realizada por Bravo y Eggerstedt ${ }^{22}$ sobre riesgos psicosociales en trabajadores (130) de diversas profesiones y ocupaciones de Lima; reportan la prevalencia de estrés laboral en 30,5\% de la población estudiada y con burnout el 14,4\%, siendo el estrés laboral el riesgo psicosocial más reconocido en los ambientes laborales y el de mayor incidencia.

Preocupados por los impactos que representan los riesgos psicosociales en la salud del trabajador, la presente investigación planteó como objetivo determinar la prevalencia del burnout y su relación con la presencia de los factores de riesgo psicosociales laborales percibidos como negativos por la población objeto de estudio de Lima.

\section{MÉTODO}

Para determinar la prevalencia del burnout y su relación con la presencia de los factores de riesgo psicosociales, se realizó un estudio descriptivo de corte transversal, correlacional, con diseño no experimental, realizado en una muestra no representativa de la Población Económicamente Activa de Lima, Perú. Se eligieron 339 trabajadores que tuvieran por lo menos un año de antigüedad laboral y que a su vez se tratara de empleados subordinados. La encuesta se aplicó a personal de diversas ocupaciones entre los que destacan obreros, oficinistas, docentes sanitaristas, etc. cuidando que no existieran más de cinco sujetos con la misma ocupación. Los instrumentos aplicados fueron: cuestionario de datos personales que incluía preguntas como edad, sexo, estado civil, años de estudio, turno de trabajo, actividad que realiza, antigüedad en la empresa y en el puesto, días de la semana trabajados, horas extras laboradas y carga horaria.

Para recabar la información relativa al burnout se utilizó el Maslach Burnout Inventory General Survey (MBI-GS de Maslach, Jackson y Leiter) ${ }^{1}$ instrumento en su nueva versión, que consta de

Tabla 1.

Caracteristicas de las variables sociales.

\begin{tabular}{clcl}
\hline \multicolumn{2}{c}{ Datos sociodemográficos } & Frecuencia & $\%$ \\
& $<37$ & 213 & 62,65 \\
& $38-47$ & 87 & 25,59 \\
Edad & $48+$ & 39 & 11,46 \\
\hline \multirow{2}{*}{ Género } & Femenino & 177 & 52,2 \\
& Masculino & 162 & 47,8 \\
\hline & Casado & 166 & 48,97 \\
& Soltero & 141 & 41,59 \\
& Viudo & 4 & 1,18 \\
& Divorciado & 8 & 2,36 \\
& Separado & 8 & 2,36 \\
Estado civil & Unión libre & 12 & 3,54 \\
\hline & 1-6 Años Básica & 4 & 1,18 \\
& 7-11 Años Media & 19 & 5,60 \\
Años de escolaridad & 12 Años o más Superior & 316 & 93,22
\end{tabular}

16 ítems y evalúa 3 dimensiones: Desgaste Emocional, Cinismo y Eficacia Profesional.

El segundo de los instrumentos fue la escala de Factores Psicosociales en el Trabajo ${ }^{2}$, que consta de 49 ítems y 7 dimensiones: condiciones del lugar de trabajo, carga de trabajo, contenido y características de la tarea, exigencias laborales, desarrollo de la carrera, interacción social y aspectos organizacionales y remuneración del rendimiento; ambos con escala tipo Likert.

Para el análisis de datos se emplearon las pruebas no paramétricas. Se utilizó el paquete estadístico Excel y SPSS, se obtuvieron frecuencias y porcentajes. Para el análisis de asociación se utilizó Chi cuadrado determinando el factor de riesgo (odds ratio OR) y los valores de $\mathrm{p}$ significativos $<0,05$.

\section{RESULTADOS}

339 participantes integraron la población estudiada; el rango de edad que predominó se ubica entre los 14 y 37 años con el 62,65\%, seguido por el rango 38 a 47 ubicándose el 25,59\%; y de 48 a 60 solo se encuentra el 11,46\%; respecto al género, el 52,2\% corresponde al femenino y 47,8\% de sexo masculino; el estado civil de la población refleja que el 48,97\% son casados, el 41,59\% solteros y el restante 9,44\% se reparte en divorciados, separados y en unión libre (Tabla 1). Referente a los datos laborales, se encontró que los años trabajados en las empresas o instituciones se caracterizan por alta rotación, representada por el 57,94\% de los trabajadores cuya antigüedad era de 1 a 2 años, el 27,94\% de 3 a 4 años y solamente el 13,82\% con antigüedad de 5 años en adelante; los años trabajados en el mismo puesto reportan el 88,82\% con una antigüedad de 6 meses y hasta 3 años es decir escasa oportunidad de ascender de puesto, el 9,71\% de 4 a 6 años y el 1,18\% más de 7 años en el mismo puesto; respecto al turno, el matutino representa el 42,77\%, seguido por el mixto con el 36,58\%, el 20,65\% se distribuye en vespertino, nocturno y jornada acumulada; las horas trabajadas por semana reportan el 70,88\% con $48 \mathrm{hrs}$ hasta $24 \mathrm{hrs}$, el 20\% y solo el 8,82\% labora más de $48 \mathrm{hrs}$, las horas extras trabajadas por semana de 1 hora el 36,87\%, 2 horas el 15,05\%, 3 horas el 35,40\% y solamente el 12,68\% no reporta trabajar horas extras (Tabla 2).

\section{Tabla 2.}

Caracteristicas de las variables laborales.

\begin{tabular}{|cccl}
\hline & Datos laborales & Frecuencia & $\%$ \\
\hline & 1 a 2 años & 197 & 57,94 \\
\hline Años trabajados & $3-4$ años & 95 & 27,94 \\
en la empresa & 5 años en adelante & 47 & 13,82 \\
\hline & 6 meses a 3 años & 302 & 88,82 \\
\hline Años trabajados en & $4-6$ años & 33 & 9,71 \\
el mismo puesto & 7 años a más & 4 & 1,18 \\
\hline & matutino & 145 & 42,77 \\
& vespertino & 16 & 4,72 \\
& nocturno & 4 & 1,18 \\
& jornada acumulada & 50 & 14,75 \\
& mixto & 124 & 36,58 \\
\hline & hasta 24 & 68 & 20 \\
Turno & 241 & 70,88 \\
Horas trabajadas & hasta 48 & 30 & 8,82 \\
\hline por semana & 48 en adelante & 43 & 12,68 \\
& 0 & 125 & 36,87 \\
\hline Horas extras trabajadas & 1 & 51 & 15,05 \\
por semana & 2 & 120 & 35,4
\end{tabular}


Sobre la exposición de factores de riesgo psicosociales, se encontró que el 10,75\% presenta una exposición alta, el 62,92\% se ubica en nivel medio; el resto, que representa el 26,33\% de los trabajadores encuestados, se encuentra en condiciones adecuadas, De los factores psicosociales estudiados, la remuneración del rendimiento y las exigencias laborales presentan mayor afectación, con un porcentaje de 31,2\% y 15,9\% respectivamente (ver Tabla 3).

Tabla 3.

Prevalencia de Factores de RiesgoPsicosociales.

\begin{tabular}{|c|c|c|c|c|c|c|}
\hline $\begin{array}{l}\text { Dimensiones de } \\
\text { los Factores Psicosociales }\end{array}$ & $\begin{array}{l}\text { Alto } \\
\text { Frecuencia }\end{array}$ & $\%$ & $\begin{array}{l}\text { Medio } \\
\text { Frecuencia }\end{array}$ & $\%$ & $\begin{array}{l}\text { Bajo } \\
\text { Frecu }\end{array}$ & cia $\%$ \\
\hline $\begin{array}{l}\text { Condiciones del } \\
\text { lugar de trabajo }\end{array}$ & 20 & 5,9 & 222 & 65,32 & 97 & 28,78 \\
\hline Carga de trabajo & 16 & 4,75 & 271 & 79,75 & 52 & 15,5 \\
\hline $\begin{array}{l}\text { Contenido y } \\
\text { caracteristicas de la tarea }\end{array}$ & 15 & 4,52 & 260 & 76,5 & 64 & 18,98 \\
\hline Exigencias laborales & 54 & 15,9 & 233 & 68,53 & 52 & 15,57 \\
\hline $\begin{array}{l}\text { Papel laboral y } \\
\text { desarrollo de la carrera }\end{array}$ & 30 & 8,9 & 232 & 68,3 & 77 & 22,8 \\
\hline $\begin{array}{l}\text { Interacción social y } \\
\text { aspectos organizacionales }\end{array}$ & 14 & 4,41 & 159 & 46,77 & 166 & 48,82 \\
\hline $\begin{array}{l}\text { Remuneración del } \\
\text { rendimiento }\end{array}$ & 106 & 31,2 & 116 & 34,12 & 117 & 34,68 \\
\hline
\end{tabular}

La Tabla 4 muestra la prevalencia del síndrome de burnout por dimensión: se reporta como Muy Alto en Desgaste Emocional o Agotamiento (DE) 6,22\% y Falta de Eficacia Profesional (EP) con 2,35\%; se ubica en Alto el Desgaste Emocional o Agotamiento con 22,7\%; en esta dimensión la prevalencia de Medio Alto con 15,59\% y la Eficacia profesional en 1,47\%; con Medio Bajo de nuevo el Desgaste Emocional o Agotamiento 13,53\%; Eficacia profesional en 3,52\%; y Cinismo (C) 1,18\%; las cifras más Bajas se ubican en Desgaste Emocional o Agotamiento 35,6\% seguido por la Falta de Eficacia Profesional con 25,88\% y Cinismo 4,7\%; respecto a la presencia Muy Bajo la reportan la Eficacia profesional, 66\%, el Cinismo con el 94\%, y el Agotamiento con 6,17\%, Lo anterior deja de manifiesto que la dimensión con mayor prevalencia del burnout es el Desgaste emocional o Agotamiento al sumar de Muy Alto a Alto el 28,85\%.

Respecto a la asociación de las variables sociodemográficas y laborales con burnout y su asociación con los factores de riesgo psicosociales, se encontró que el género presentó asociación con la falta de eficacia profesional $(\mathrm{p}=0,005)$, desgaste emocional $\mathrm{o}$ agotamiento $(p=, 001)$ y cinismo $(p=0,51)$; de igual manera esta variable se relacionó con los factores psicosociales en las dimensiones condiciones del lugar de trabajo $(p=0,000)$, el papel y desarrollo de la carrera $(\mathrm{p}=0,000)$, y remuneración y rendimiento $(\mathrm{p}=0,000)$; la edad no reportó asociación al burnout; con las condiciones del lugar de trabajo se identificó $(\mathrm{p}=0,025)$ y exigencias laborales $(p=0,034)$; la variable estado civil con el desgaste emocional o agotamiento $(\mathrm{p}=0,000)$; esta misma presentó asociación con las 7 dimensiones de los factores psicosociales; $(p=0,048)$
Tabla 5.

Asociación de las variables sociodemográficas y laborales con el Burnout y los Factores de Riesgo Psicosociales.

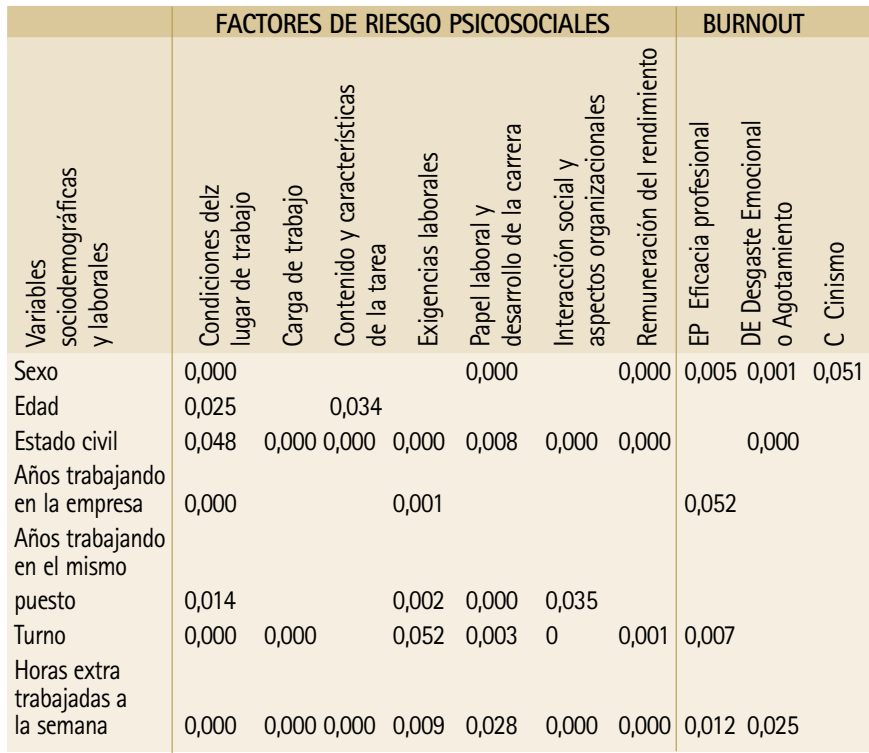

con las condiciones del lugar de trabajo $(p=0,000)$ con la carga de trabajo, contenido y características de la tarea y exigencias laborales, interacción social y aspectos organizacionales y remuneración del rendimiento, y $(\mathrm{p}=0,008)$, en la dimensión papel y desarrollo de la carrera; los años trabajando en la empresa presentaron asociación con la eficacia profesional $(p=0,052)$, asimismo con las condiciones del lugar de trabajo $(p=0,000)$, y las exigencias laborales $(p=0,001)$; por su parte los años trabajando en el mismo puesto no reportaron asociación al burnout, a diferencia con los factores psicosociales la dimensión condiciones del lugar de trabajo ( $p=0,014)$, las exigencias laborales $(p=0,002)$, papel laboral y desarrollo de la carrera $(\mathrm{p}=0,000)$, interacción social y aspectos organizacionales $(p=0,035)$; el turno se asocia con la eficacia profesional ( $\mathrm{p}=0,007)$, esta misma variable con las condiciones del lugar de trabajo $(\mathrm{p}=0,000)$, carga de trabajo $(\mathrm{p}=0,000)$, papel laboral y desarrollo de la carrera $(\mathrm{p}=0,003)$, interacción social y aspectos organizacionales $(\mathrm{p}=0,000)$, remuneración del rendimiento $(p=0,001)$; finalmente las horas extras trabajadas a la semana se asocian con la eficacia profesional $(p=0,012)$, desgaste emocional o agotamiento $(\mathrm{p}=0,025)$ al igual la variable reportó asociación con los factores psicosociales en las 7 dimensiones $(p=0,000)$ con las condiciones del lugar de trabajo, carga de trabajo, contenido y características de la tarea, interacción social y aspectos organizaciones y remuneración del rendimiento $(p=0,000)$, exigencias laborales $(\mathrm{p}=0,009)$ y papel laboral y desarrollo de la carrera $(p=0,028)$, Lo anterior evidencia que existe asociación significativa entre los factores psicosociales y las variables sociodemográficas y laborales, sexo, estado civil, años trabajando en el

Tabla 4. Prevalencia del Burnout.

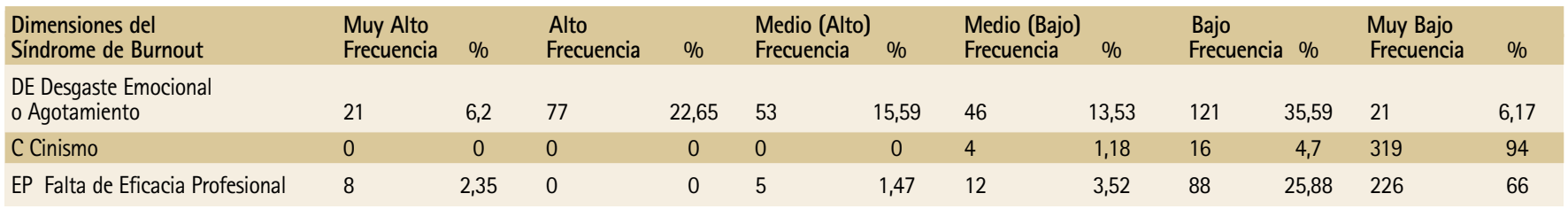


mismo puesto, turno y las horas extras trabajadas; de igual manera estas con el burnout a excepción de los años trabajados en el mismo puesto, como se presenta en la Tabla 5.

En la Tabla 6 se identifican los factores de riego psicosociales asociados al burnout, se encontró que las malas condiciones del lugar de trabajo resultaron ser un factor de riesgo para la presencia del cinismo con $\mathrm{OR}=3,603$; el contenido y caracteristica de la tarea afectaron la eficacia profesional, $\mathrm{OR}=4,517$; de igual manera la carga de trabajo incidió en la eficacia profesional con $\mathrm{OR}=5,896$; por su parte las exigencias laborales se vincularon con el cinismo $\mathrm{OR}=6,979$; en tanto que el papel laboral y desarrollo de la carrera mantuvieron asociación con el agotamiento, $\mathrm{OR}=2,392$; asimismo, la inadecuada interacción social y aspectos organizacionales impactaron en el agotamiento $\mathrm{OR}=2,705$ y cinismo $\mathrm{OR}=$ 2,705; finalmente la remuneración del rendimiento presentó asociación con el cinismo $\mathrm{OR}=2,965$, Los factores psicosociales de mayor riesgo y asociación significativa al burnout fueron las exigencias laborales, la carga de trabajo, el contenido y características de la tarea y las condiciones del lugar de trabajo.

Tabla 6.

Factores de Riesgo Psicosociales asociados al Burnout.

\begin{tabular}{|c|c|c|c|}
\hline $\begin{array}{l}\text { Factores } \\
\text { psicosociales }\end{array}$ & $\begin{array}{l}\text { EP Eficacia } \\
\text { Profesional }\end{array}$ & $\begin{array}{c}\text { DE } \\
\text { Agotamiento }\end{array}$ & $\stackrel{\text { C }}{\text { Cinismo }}$ \\
\hline $\begin{array}{l}\text { Condiciones del } \\
\text { lugar de trabajo }\end{array}$ & & & $\begin{array}{c}p=, 006 \\
O R=3,602 \\
(3,031-4,281)\end{array}$ \\
\hline Carga de trabajo & $\begin{array}{c}p=, 022 \\
O R=5,896 \\
(1,426-24,374)\end{array}$ & & \\
\hline $\begin{array}{l}\text { Contenido y } \\
\text { caracteristicas de la tarea }\end{array}$ & $\begin{array}{c}p=, 045 \\
O R=4,517 \\
(1,098-18,571)\end{array}$ & $\begin{array}{c}p=, 003 \\
O R=2,219 \\
(1,277-3,855)\end{array}$ & \\
\hline Exigencias laborales & & & $\begin{array}{c}p=, 004 \\
O R=6,979 \\
(5,371-9,068)\end{array}$ \\
\hline $\begin{array}{l}\text { Papel laboral y } \\
\text { desarrollo de la carrera }\end{array}$ & & $\begin{array}{c}p=, 001 \\
O R=2,392 \\
(1,425-4,015)\end{array}$ & \\
\hline $\begin{array}{l}\text { Interacción social y } \\
\text { aspectos organizacionales }\end{array}$ & & $\begin{array}{c}p=, 008 \\
O R=1,750 \\
(1,131-2,705)\end{array}$ & $\begin{array}{c}p=, 056 \\
O R=2,068 \\
(1,851-2,310)\end{array}$ \\
\hline $\begin{array}{l}\text { Remuneración } \\
\text { del rendimiento }\end{array}$ & & & $\begin{array}{c}p=, 014 \\
O R=2,965 \\
(2,551-3,445)\end{array}$ \\
\hline
\end{tabular}

\section{DISCUSIÓN}

Estudios realizado en personal de enfermería del Perú, reportan prevalencia baja de burnout ${ }^{23}$; en el estudio realizado en el área de emergencias del hospital nacional Guillermo Almenara Irigoyen, se encontró 13,6\% en la dimensión de despersonalización y niveles medios en las dimensiones de agotamiento emocional $(31,8 \%)$ y despersonalización $(36,4 \%)$; niveles más bajos en las tres dimensiones del burnout son los referidos por Gamonal, García y Silva ${ }^{24}$ en su investigación en unidades críticas de una institución de salud infantil: en la dimensión despersonalización 80,0\%, cansancio emocional 66,7\% y realización personal 65,7\%.

La prevalencia del sindrome de burnout en profesionistas de la educación básica en Arequipa, Perú, reportan que el 93,7\% de los profesores presentaron un nivel moderado, ubicándose el 6,3\% en nivel severo ${ }^{25}$; estos hallazgos difieren con lo referido por Fernández Arata $^{21}$, al encontrar la presencia de burnout en niveles elevados, manifestando que fue más alto en los docentes de escuela primaria $(43,2 \%)$ que en los de secundaria $(36,6 \%)$ encontrando una relación significativa entre los estresores y el burnout. La encuesta aplicada a 130 trabajadores de diversas profesiones y ocupaciones en Lima manifiesta la prevalencia de burnout en $14,4 \%$, siendo el estrés laboral el riesgo psicosocial más reconocido en los ambientes laborales y el de mayor incidencia ${ }^{22}$; es importante destacar que los autores que elaboraron la citada encuesta no señalan los criterios empleados para identificar a quienes tienen burnout.

Estudios en los que se aplicó el MBI-GS en trabajadores que desempeñan ocupaciones no asistenciales refieren cifras de prevalencia del burnout de muy alto en la dimensión eficacia profesional (EP) y desgaste emocional o agotamiento (DE); la prevalencia del burnout reportada en el presente difieren, en la eficacia profesional se encontró como muy alto solamente el 2,36\%; datos similares en desgaste emocional o agotamiento emocional al reportar el 6,22\%; presencia muy baja en cinismo con 94\% y falta de eficacia profesional 66\%; estos datos son menores que los reportados en un estudio realizado con trabajadores de la industria manufacturera, el que encontró presencia muy alta en las dimensiones eficacia profesional $(8,3 \%)$ y agotamiento emocional $(4,6 \%)$ y en ambos casos, coincide con baja presencia en cinismo ${ }^{26}$; por su parte, los hallazgos del estudio realizado por Valdivia $^{27}$ en conductores de microbuses de la ciudad de Santiago de Chile difieren aun más con los del presente estudio, revelando la presencia del síndrome, caracterizado por niveles bajos en cinismo 56,67\%, pero altos en desgaste emocional o agotamiento $45,83 \%$ y eficacia profesional 68,33\%; la prevalencia que reporta el estudio realizado en una empresa constructora de Maracaibo, difiere incluso más con la prevalencia encontrada en nuestro estudio, al ubicar la presencia de muy alto en la eficacia profesional con 34,09\%, en tanto que el desgaste emocional o agotamiento y el cinismo se ubican de bajo a muy bajo con $54,54 \%$; y $45,45^{28}$.

Los hallazgos del presente estudio evidenciaron que el burnout está asociado a la presencia de factores de riesgo psicosociales percibidos como negativos por la población económicamente activa de Lima; el mayor factor de riesgo lo representan las exigencias laborales que inciden en el cinismo, seguido de la carga de trabajo que impacta la eficacia profesional, esta misma con el contenido y las características de la tarea carga de trabajo, y las condiciones del lugar de trabajo con el cinismo; lo anterior difiere con lo reportado por Aranda, López y Barraza ${ }^{5}$ en su investigación realizada con trabajadores de la industria de la transformación de la masa al encontrar únicamente asociación significativa entre los factores psicosociales y el síndrome en la dimensión agotamiento emocional con el área de sistema de trabajo; por su parte el estudio efectuado por Pando, Castañeda, Gómez, Águila, Ocampo y Navarrete ${ }^{29}$ en docentes de la Universidad del Valle de Atemajac, Guadalajara, México, encontraron asociación entre agotamiento y la carga de trabajo, características de la tarea, y desarrollo de la carrera, despersonalización y desarrollo de la carrera, la eficacia profesional con el desarrollo de la carrera; si bien las diferencias con el último estudio pueden deberse en gran medida a la actividad asistencial que desempeñan estos trabajadores, y el nuestro no.

El estudio referido líneas arriba realizado por Arias y Jiménez ${ }^{25}$ en 
docentes de educación básica en Arequipa, coincide con nuestros hallazgos $(\mathrm{p}<0,004)$ respecto a la asociación encontrada entre los factores de riesgo psicosocial y el burnout, al reportar relaciones significativas $(\mathrm{p}<, 000)$ entre el tipo de gestión educativa y la despersonalización y la baja realización personal y el burnout.

A la luz de los resultados obtenidos en el presente estudio, se concluye que la prevalencia del sindrome de burnout de los trabajadores que representaron la población económicamente activa de Lima, Perú, es de baja a media en el 93,82\%, en tanto que en el $6,22 \%$ se ubica en alta; asimismo se confirma que este sindrome no es exclusivo de profesiones y ocupaciones asistenciales en virtud de los hallazgos antes expresados; de igual manera se manifiesta que existe asociación significativa entre el burnout y la presencia de factores de riesgo psicosociales percibidos como negativos por los trabajadores.

Por lo anterior se recomienda a las empresas y organizaciones emprender medidas y estrategias para el establecimiento de políticas que promuevan la salud en el trabajo a través de la aplicación de las normas vigentes en materia de factores de riesgo psicosocial, que minimicen los daños a la salud como el burnout que afecta física y mentalmente a los trabajadores, la productividad de la empresa y los gastos en atención de la salud.

\section{REFERENCIAS}

1. Maslach C, Jackson SE, Leiter M, Maslach Burnoutl nventory, 3rd ed, Palo Alto: Consulting Psycologist Press; 1996.

2. Silva GN, Factores psicosociales y desgaste profesional en académicos del Centro Universitarios de Biológicas Agropecuarias [tesis de doctorado], Jalisco: Universidad de Guadalajara; 2006.

3. Organización Internacional del Trabajo, Factores psicosociales en el trabajo: naturaleza, incidencia y prevención, Informe del Comité Mixto OIT/OMS sobre medicina del trabajo, Ginebra: OIT-OMS; 1984.

4. European Agency for Safety and Health at Work, Office for Official Publications of the European Communities, Researchonwork-related stress [on line], Luxembourg: EU-OSHA; 2000 [citado 19 oct. 2014] Disponible en: http:osha,europa,eu/en/publications/reports/203.

5. Aranda BC, López GJL, Barraza SJH, Factores psicosociales y síndrome de burnout en trabajadores de la industria de la transformación de la masa, Tepic, México, Rev Col Psiquiatr, 2013; 42(2):167-172.

6. Marrero ML, Román JJ, Salomón AN, Estrés psicosocial como factor de riesgo para las complicaciones de la gestación y bajo peso al nacer, Rev Cubana Salud Pública [en línea] 2014 [citado 20 oct 2014]; 39(1): 864-880,Disponible en: http://scielo,php?script=sci_arttext\&pid=S0864-3466213000500006\&lng=e s\&nrm=iso.

7. Gil-Monte PR,Peiró JM, Desgaste psíquico en el trabajo:el síndrome de quemarse, Madrid: Sintesis; 1997.

8. Freudenberger HJ,Burnout, J Social Issues, 1974;30:159-165.

9. Maslach C, Jackson SE,Maslach Burnout Inventory, Research Edition, Palo Alto California: Consulting Psychologist Press; 1981.

10. Maslach C, Jackson SE, Burnout in health's professions: A social psychological analysis, En: Sanders G,SulsJ, Social Psychology of Health and Illness, Hillsdale: Lawrence Erlbaum; 1982.

11. Maslach C, Jackson SE,The Maslach Burnout Inventory Manual,Palo Alto, California: Consulting Psychologist Press; 1986.

12. Proccini J, Kiefaber MW, Parent burnout, New York: Doubleday; 1983,

13. Pines $A M$, Keeping the spark alive: Preventing burnout in love and marriage, New York: St Martin Press; 1988.

14. Moreno JB, Rodríguez CR, Escobar RE, La evaluación del Burnout Profesional, Factorialización del MBI-GS, Un análisis preliminar, Ansiedad y Estrés, 2001; 7(1):69-78.

15. Bandura A, Self-efficacy: Toward a unifying theory of behavioral change, Psychol Rev, 1977; 84:(2):191-215.

16. Gil-Monte $P$, Validez factorial de la adaptación al español del Maslach Burnout Inventory-General Survey, Salud Pública Méx, 2002; 44(1):33-40.

17. Aranda BC, El Sindrome de Burnout o de quemarse en el trabajo: un conflicto en la salud de los profesionales, Revista de investigación en psicología (Lima), 2011; 14(2):47-56.
18. Organización Internacional del Trabajo, La Prevención de las Enfermedades Profesionales, Ginebra: 0IT; 2013.

19. Organización Internacional del Trabajo, Guia del Formador SOLVE; Integrando la promoción de la salud a las políticas de Salud y Seguridad en el Trabajo(SST), 2a, ed, Ginebra: OIT; 2012.

20. Raffo E, Ráez L, Cachay 0, Riesgos psicosociales, Revista de la Facultad de Ingenieria Industrial, 2013;16(2):70-79.

21. Fernández $M$, Burnout, autoeficacia y estrés en maestros peruanos: tres estudios fácticos, CiencTrab [en línea] 2008; 30(1):120-125 [citado 21 oct 2014], Disponible en: http://www,cienciaytrabajo,cl/cyt/Paginas/EdicionesAnteriores,aspx

22. Bravo $E$, Eggerstedt $M$, Los Riesgos psicosociales en los ambientes laborales Peruanos [en línea]2014 [citado 21 oct 2014], Disponible en:http://www, infocapitalhumano,pe/articulos, php?id=561\&t=los-riesgos-psicosocialesen-los-ambientes-laborales-peruanos.

23. López C, Zegarra A, Cuba V, Factores asociados al Sindrome de Burnout en Enfermeras de emergencia del hospital nacional Guillermo Almenara Irigoyen, Rev Ciencias de la Salud [en línea]2006 [citado 21 oct 2014];(1):1,Disponible en: http://posgrado,upeu,edu,pe/revista/file/5563, pdf.

24. Gamonal Y, García C, Silva M, Sindrome de Burnout en el profesional de enfermería que labora en áreas criticas, Rev enferm Herediana [en línea]2008 [citado 21 oct 2014];01(1):33-39, Disponible en: http://www,upch,edu,pe/faenf/images/pdf/rev20125,2/ VOL11/ v1n1a05,pdf.

25. Arias W, Jiménez N, Síndrome de burnouten docentes de Educación Básica Regular de Arequipa, Educación (Perú) [en línea] 2013 [citado 20 oct 2014];42:53-76, Disponible en: http://revistas,pucp,edu,pe/index,php/ educacion/article/viewFile/5291/5288

26. Dávila $M, C l i m a$ organizacional y Sindrome de Burnout en una empresa mediada de manufactura, TES Coal, 2011; 32:1-9.

27. Valdivia $H$, Rojas $F$, Marin $M$, Montenegro J, León $C$, Yévenes $R$, Díaz $V$, Sindrome de Burnout en conductores de microbuses de la ciudad de Santiago de Chile, Rev, Chil, Stud, Med, 2012; 6(1):25-30.

28. Unda C, Agotamiento emocional en empleados de una empresa constructora [tesis de maestría] [en línea], Maracaibo: Universidad Rafael de Urdaneta, Facultad de Ciencias Políticas, Administrativas y Sociales, Escuela de Psicologia; 2009, 93 p[citado 20 oct 2014], Disponible en: http://200,35,84,131/portal/bases/marc/texto/3201-09-02883,pdf.

29. Pando MM, Castañeda TJ, Gregoris GM, Águila, MA, Ocampo L, Navarrete $R R$, Factores psicosociales y sindrome de burnout en docentes de la Universidad del Valle de Atemajac, Guadalajara, México, Salud en Tabasco, 2006;12:(3):523-529. 